\title{
Trauma Healing Club: Utilizing Culturally Responsive Processes in the Implementation of an After-School Group Intervention to Address Trauma Among African Refugees
}

\author{
Susan Elswick ${ }^{1}$. Gregory Washington ${ }^{1} \cdot$ Hannah Mangrum-Apple ${ }^{1} \cdot$ Christy Peterson $^{1} \cdot$ Ebony Barnes $^{1}$. \\ Paige Pirkey ${ }^{1}$. Jerry Watson ${ }^{1}$
}

Accepted: 1 July 2021 / Published online: 24 July 2021

(c) The Author(s), under exclusive licence to Springer Nature Switzerland AG 2021

\begin{abstract}
The State of Tennessee granted appropriations to an urban university in the West Tennessee region to address the needs of at-risk African refugee children and families who have experienced Adverse Childhood Experiences (ACEs). Action oriented research efforts were conducted to address the needs of youth in the local region by developing, implementing, and evaluating a community-based intervention that was not only trauma responsive but culturally competent. The Trauma Healing Club was a treatment package developed and researched for this purpose and offered in an after school support program. This identified intervention utilized an existing evidence-based trauma intervention framework that was adapted to include more culturally responsive processes such as pyramid mentoring and African drumming. This work aimed to evaluate the effectiveness of an integrated trauma-informed practice model for community-based programming and the effectiveness of adapting an existing intervention to better fit the community needs. Results indicated that the adaptation of the traumaresponsive intervention was effective and supportive of the child-participant and his/her family needs -both culturally and as it relates to improved participant functioning post intervention.
\end{abstract}

Keywords Group intervention $\cdot$ Trauma $\cdot$ Evidence-based $\cdot$ Culturally responsive $\cdot$ Refugees

\section{Introduction}

When identifying needs of students within our local school community, often, refugee children and families are often overlooked. This specific subset of the student population

Susan Elswick

selswick@memphis.edu

Gregory Washington

gwshngt1@memphis.edu

Hannah Mangrum-Apple

hmangrum@memphis.edu

Christy Peterson

cpetrsn2@memphis.edu

Ebony Barnes

ejbarnes@memphis.edu

Paige Pirkey

Paige.Pirkey@memphis.edu

Jerry Watson

Jerry.Watson@memphis.edu

1 School of Social Work, University of Memphis, Memphis, TN, USA may need trauma-responsive interventions more than their natural born citizen counterparts. Over the last few decades, the United States has become a safe haven for many African refugees seeking solace from the adverse experiences often found in their home countries such as civil unrest and war, extreme poverty, community violence, religious persecution, and even unexpected natural disasters (Adedoyin et al., 2016). Per report, there are currently close to 80 million forcibly displaced people worldwide, which is a drastic rise since the 16.2 million of whom were reported as displaced during 2017 (UNHCR, 2020). The COVID-19 pandemic exacerbated the issues with forcibly displaced individuals globally (UNHCR, 2019). In addition to these aforementioned traumatic experiences, many African refugees experience continued trauma during the process of seeking asylum and even in the acculturation and assimilation experiences they have once safely in the United States. For example, African refugees can spend weeks if not years in refugee camps awaiting approval and safe travel into the United States. The separation from family, in addition to less than stable and nurturing camp environments, can be identified as a continued stressor in their lives (Ramsden \& Taket, 2013). Then, once approved for 
relocation to the United States, many African refugee families must face the impact that the Western culture will have on their family unit (Miller \& Rasmussen, 2010). African refugee families often find themselves faced with issues of acculturation and assimilation over time. Acculturation is a process of cultural exchange through which a person or group adopts certain values and practices of a new culture that is not originally their own, in an attempt for survival (Schwaartz et al., 2010). The result of acculturation is that the original culture of the person remains, but it is changed greatly by this process. Assimilation is the process by which a person or group adopts the new culture in which they are embedded and this new culture replaces their original culture, leaving only small parts of the original culture to be evident (Schwartz et al., 2010).

Per report, many first-generation immigrants often engage in the process of acculturation when adapting to a new community to succeed socially and economically (Cole, 2019). Many immigrants across the nation are often compelled by local and regional laws to acculturate, and then eventually assimilate. Due to these requirements, many refugees must learn not only the native language but also the local laws of their new community and broader society (Cole, 2019). The challenge and cost of acculturation and assimilation can be great for many refugees and immigrant populations. Because of the potential impact that these life transitions can have on refugee children and families, school-based practitioners must begin to assess the levels of trauma for families who are in these situations and provide effective traumaresponsive interventions for these families. This article will review the importance of understanding the cultural needs of this student population, and describe an effective traumaresponsive intervention that embeds cultural supports that can be replicated by school-based practitioners in the field.

\section{Need for Trauma Informed Programming in this Region}

To understand the need for such a comprehensive and adaptive trauma intervention, evaluating local statistics of the identified region, as well as the statistics and needs of the identified population, African refugees, is necessary.

As previously mentioned, many African refugees have numerable adverse experiences prior to ever entering the United States. Some studies indicate that African refugees have suffered more traumatic experiences than other refugees from other parts of the world (Hooberman et al., 2007; Rousseau et al., 2001). In a study conducted by Jaranson et al. (2004), African refugees were reported as being four times as likely to have an incidence of PTSD than any other refugee groups. The compounding factors of these identified traumatic experiences faced by African refugees from their home country, the experiences they have in attempting to get into the United States, and finally, issues of assimilation and acculturation significantly predisposes African refugees to be symptomatic of posttraumatic stress disorder (PTSD) and other mental health disorders (Akinsulure-Smith, 2012; Matheson et al., 2008).

Once established in the United States, many refugees frequently experience social inequities and health disparities that are related to limited access to needed resources, health-related issues, and loss of meaningful social roles and social support. In addition to these issues, African refugees must also face racism, discrimination, and marginalization of their cultural practices. Also, many of these refugees are relocated to areas and regions of the United States that are thought to be more representative and supportive of the refugee group; however, many of these identified locations have their own struggles with adversity that only continue the cycle of trauma exposure for these refugee families.

For example, the proposed trauma intervention was developed for a large urban area in West Tennessee specifically focusing on the African refugee population for after school supports. In addition to the identified needs of the African refugee population locally, it is also important to understand the regional statistics on ACEs for these families. According to the 2017 Census data, the specific area has a population of $1,346,437$ with an overall poverty rate of $19.4 \%$ and a child (under 18) poverty rate of $30 . \%$. The population of the region is $46 \%$ African American, 44\% non-Hispanic white, and 5.3\% Hispanic, although the latter population is increasing rapidly (Delavega, 2020).

This region experiences a significant number of risk factors related to mental health that impact both children and adults living in the region. Poverty is an important and incredibly relevant factor. This West Tennessee area continues to be the poorest metropolitan area with a population exceeding 1,000,000 (Delavega, 2020). Poverty in the identified area disproportionately impacts African Americans and Latinos, with $28.3 \%$ and $42.8 \%$ rates, respectively (Delavega, 2020). Poverty pervades all aspects of life, impacts students, and is highly correlated to race. Schools where $80 \%$ or more of the student population is minority are the same schools where $80 \%$ or more of the students live in poverty (Delavega, 2020). More than sixty percent (60.4) of children living in the region live in a home headed by a single parent (The Urban Child Institute, 2013).

Additionally, West Tennessee falls significantly below national averages in population health. African Americans in the region experience the highest rates of sexually transmitted diseases compared to the rest of the United States (Urban Child Institute, year). This area has a 34\% obesity rate which ranks 83 rd out 95 Tennessee counties in terms of health factors per the Centers for Disease Control and Prevention (2017). To make matters worse, this region has been designated a Health Professional Shortage area by 
the U.S. Department of Health and Human Services due to having a partial low-income population (Melton, 2017). Unsurprisingly, all of the counties of West Tennessee are designated as Medically Underserved Areas (MAUs) or Federal Primary Care Shortage Areas for Mental Health (Tennessee Department of Health, 2017).

In addition to the many adversities that local families face within our region, there is a growing body of research that has made it increasingly apparent that adverse childhood experiences (ACEs) are a critical public health issue. ACEs are potentially traumatic experiences and events, that range from abuse and neglect to living with an adult with a mental illness. The extant literature on the negative outcomes of ACEs on child development and future health outcomes has been repeatedly substantiated over time. Data collected in Tennessee in 2016 and 2017, indicated that $61 \%$ of study participants reported at least $1 \mathrm{ACE}$, and $27 \%$ reported experiencing 3 or more ACEs (Tennessee Department of Health, 2017). Additionally, national statistics indicate that child neglect accounts for $78 \%$ of the child maltreatment cases in the nation. Although child maltreatment can include physical, medical, and educational neglect, emotional neglect is one of the most common forms of maltreatment (e.g., inattention to a child's emotional needs or psychological care or letting the child use alcohol or drugs). The need for a supportive intervention that addresses ACEs as a public health issue from a supportive parental approach and perspective is needed.

There is growing interest in understanding the prevalence of these experiences across different communities in the United States, and how to prevent and respond to them. One mechanism responsible for these effects-toxic levels of stress-can be substantially buffered by a stable and supportive relationship with a caregiver. Also, keeping in mind that refugee families may experience ACEs at a much higher rate that other families within the regional community, and that ensuring culturally relevant processes are embedded in the trauma work is imperative to success. The Trauma Healing Club intervention was developed and implemented for this region to focus on offering promotion activities, prevention activities, and direct intervention to children, families, and community partners in the local region and beyond. The Trauma Healing Club utilized culturally sensitive processes when implementing a wellknown trauma responsive intervention.

\section{Need for Culturally Sensitive Trauma Informed Practices}

The African refugee population is identified as an understudied and underserved population within the area of trauma research and intervention (Akinsulure-Smith, 2012; Filippi et al., 2014; Guenther et al., 2012). There is a need for more intensive research about the trauma-based interventions that are deemed successful for this marginalized and forgotten population. Mental health interventions used with African refugees have been primarily trauma-focused, but the results of the effectiveness of the interventions on the African refugee population have been mixed (Carlsson et al., 2005). Additional research on effective, culturally responsive, evidence-based interventions for addressing trauma must be conducted. Valuing refugees' cultural knowledge and experience is an important part of trauma-based interventions, because it assists in recreating valued social roles and enables participants to maintain their cultural connections and identity (Goodkind et al., 2014).

Culturally sensitive trauma-informed care requires attention to familial and child values and beliefs regarding mental health and wellness (Meléndez Guevara et al., 2021). Additionally, what is unique from other interventions is that trauma-informed processes that are culturally sensitive pay specific attention to cultural variations in the child's and family's experience of and response to trauma (Yeager \& Bauer-Wu, 2013). Research suggests that although there is a universal biological response to trauma, cultural factors can influence the biopsychosocial experience of trauma and subsequent traumatic stress reactions (Whaley \& Davis, 2007). Trauma-informed clinicians need to be aware thar ethnocultural factors play an important role in an individual's vulnerability to, and experience and expression of traumatic stress, as well as one's response to trauma treatment (TrepassoGrullon, 2012). Because of these aforementioned factors, the research team for this work decided to make some adaptations to an existing, evidence-based trauma intervention so that it took into account the need for additional activities to engage the participants in the healing process. School-social workers need to be prepared to adapt interventions to be more focused on cultural relevance of the populations that they are serving (Johnson \& Munch, 2009; Kohli et al., 2010; Kontak, 2012). The following paragraphs will describe how an existing intervention was adapted to meet the needs of the children in a supportive after school program.

\section{Trauma Healing Club Intervention}

Based on the prior review delineating the African refugee population being high-risk for negative effects of continued and long-term adversity, and the need for culturally-sensitive trauma responsive interventions, this research team developed a treatment package and intervention known as The Trauma Healing Club (THC) in an attempt to incorporate the refugee family's needs and culture into an existing evidencebased trauma intervention. In the following paragraphs, how the evidence-based trauma intervention, Cognitive Behavioral Interventions for Trauma in Schools (CBITS), was 
adapted and enhanced using parent and participant feedback, as well as culturally sensitive processes, will be described.

Grounding the Trauma Healing Club in evidence-based practice was foundational for this treatment package and intervention. The Trauma Healing club utilizes the 10-week CBITS framework and then, utilizes culturally responsive programming to adapt that intervention to the African refugee population. CBITS was developed by a team of clinician-researchers from the RAND Corporation, the University of California at Los Angeles (UCLA), and the Los Angeles Unified School District (LAUSD) (Jaycox et al., 2012). CBITS is a school-based, group and individual intervention. It is designed to reduce symptoms of post-traumatic stress disorder (PTSD), depression, and behavioral problems, and to improve functioning, grades, and attendance, peer and parent support, and coping skills (Jaycox et al., 2012). CBITS has been effectively used with students from 5 th through 12th grade who have witnessed or experienced traumatic life events. CBITS utilizes a cognitive-behavioral intervention and approach to practice, which has been extensively researched since 2000; results consistently show that students who participate in the program have significantly fewer reported symptoms of post-traumatic stress, depression, and psychosocial dysfunction (Jaycox et al., 2012). The CBITS intervention is a solid foundation to embed more culturally relevant practices for the African refugee population. Within this research, and for The Trauma Healing Club, small adaptations to the intervention were made to maintain the fidelity of the evidence-based intervention while also meeting the needs of the specific population being served.

One specific adaptation was the addition of African drumming in each session as a way in which participants could regulate and process their emotions during the intervention. There are a number of studies that show the positive effect that drumming has on social-emotional, behavioral health, and well-being of at-risk youth (Ho et al., 2012; Bensimon et al., 2008; Clift, 2013; Fancourt et al., 2016a, b; Reagon et al., 2016). Additional studies on groupbased music processes suggest that drumming has many benefits, including increased self-confidence (Matarasso, 1997), improved health and wellbeing (Clift, 2013; Fancourt et al., 2016a, b; Reagon et al., 2016), and enhanced social connections and supports (Richman, 1993). Research has also indicated that group music encourages social bonding for specific groups (Pearce et al., 2016; Weinstein et al., 2016). Notably, African drumming is significant for African refugees (Vinesett et al., 2015). Previous research has shown that the use of African drumming in mental health programming for African refugees resulted in participants describing diverse benefits such as increased exercise tolerance (Faulkner et al., 2012), stress reduction (Hars et al., 2014), feelings of group support (Fancourt et al., 2016a, b), and beneficial spiritual experiences (Hays \& Minichiello,
2005). Because of this notable research and findings, African drumming was an added part to the Trauma Healing Club intervention.

Another adaptation made to the intervention was the addition of a Pyramid Mentoring process. Pyramid Mentoring is designed to contribute to cultural socialization, cultural identity, and cognitive development through the provision of modeling and social support. A key component is culturally centered, psycho-educational learning utilizing multiple generations of elders in a triangle or pyramid of social interaction. This approach incorporates the strengths of group mentoring that have shown promise in allowing youth to get various perspectives on the pathways to healthy adult development in part because of the integrated role play strategies for avoiding unhealthy behavior (Washington et al., 2007, 2014). For the purposes of this intervention, Pyramid Mentoring was used as a model for student participants who successfully completed The Trauma Healing Club intervention and had a post-test CPSS score below the inclusion criteria of 14 . The successful student graduates are then trained in mentoring processes so that they can support the next cohort of Trauma Healing Club participants in healing from their past trauma. The inclusion of peer-based pyramid mentoring was added after a significant number of The Trauma Healing Club graduates from cohort 1 asked to continue to participate in the intervention although they were no longer clinically in need of services. Additionally, utilizing a peer mentoring component to this work supports the evidence that peer support led programs has a reciprocal effect on participants and peers (Chinman et al., 2014, 2015; Corrigan \& Sokol, 2013). With the addition of African drumming and the pyramid mentoring process, the Trauma Healing Club treatment package was developed. The following table (Table 1) supports the reader in understanding how we took an evidence-based practice model and made it more culturally responsive and representative of the African refugee population we were serving.

The final adaptation made to the CBITS curriculum for The Trauma Healing Club, was to extend the original 10 -week curriculum to a 12-week curriculum so that we had time to add in the drumming component of the intervention without the risk of losing the content covered in the 10-week CBITS intervention. To address this addition of drumming activities, The Trauma Healing Club team developed an adjunct manual to coordinate with the existing CBITS manual. The purpose of the adjunct manual was to ensure that culturally relevant African programming and drumming was effectively added into the intervention. This adjunct manual embedded drumming activities into the curriculum which also included drum ceremony activities; due to the addition of drumming activity content, an additional 2 weeks was added to the curriculum making this a 12-week curriculum in total. 


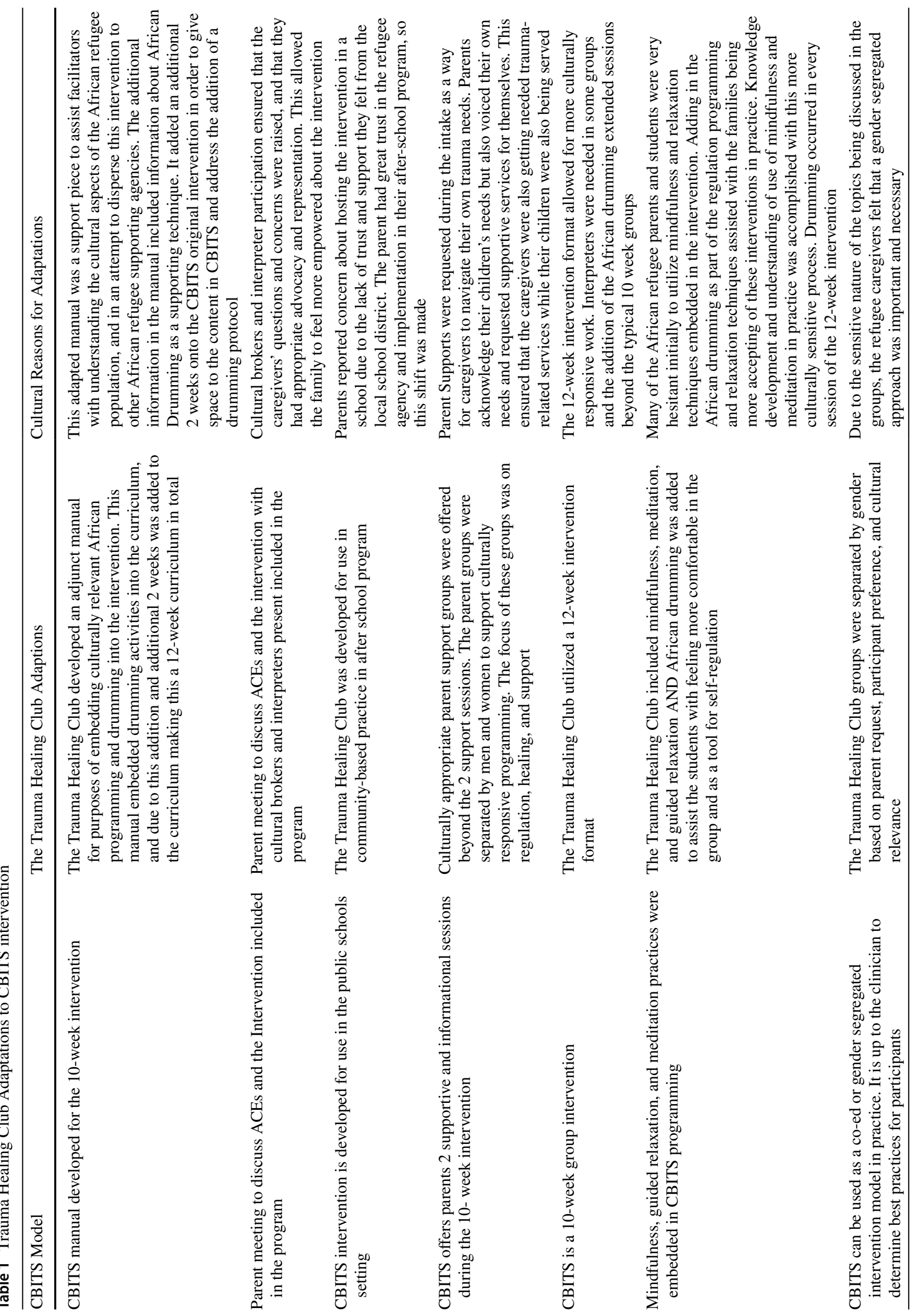


To best serve the community partnering agency and the population's needs, researchers ensured that the Trauma Healing Club intervention would run in the Fall and Spring semesters to work around school and family schedules. The initial Trauma Healing Club launched in September of the year the intervention was implemented and the first cohort completed their intervention by the end of November. This allowed for the intervention to be offered to multiple students within the first year of service delivery. The timeline and process for implementing the Trauma Healing Club Intervention depicted below in Table 2 .

\section{Method}

The researchers obtained appropriate approvals through their institutional review board (IRB) prior to beginning this research. In addition to the IRB approval, parental consent and participant assents were obtained during the introductory psychoeducational training sessions. The researchers utilized a mixed methods multiple time series research design to examine change in participants behaviors, symptoms, and outcomes after participating in the Trauma Healing Club intervention. The researchers utilized both qualitative and quantitative data to support the outcomes and future work. The research team utilized screening tools for African refugee caregivers and their boys' and girls' being served in the treatment groups who were ages 12-18 at the local refugee support agency during their after-school program.

\section{Participants}

The community partner and program utilized for this work served African refugee families in the local area. The community partner assisted in the identification of potential participants based on behavioral needs, and incentives were offered to the student and families three times during the 12 -week intervention. The family received a $\$ 50$ amazon gift card three different times in order to support the family's willingness and motivation to sustain participation in the 12-week intervention. The student participants received snacks during each trauma intervention session, and they received their own djembe drum at the conclusion of the intervention as a graduation present from the 12-week trauma intervention. This recruitment and incentive package yielded the participation of 88 student participants (51 males and 37 females).

Consent and assent for this specific trauma intervention were obtained prior to beginning the intervention. Parents of identified students were provided informed consent and were oriented to the program. The parents were provided a psychoeducational training on the Adverse Childhood 
Table 2 Trauma Healing Club Timeline and Process

\begin{tabular}{|c|c|}
\hline $\begin{array}{l}\text { Month of Year in Interven- } \\
\text { tion }\end{array}$ & Activity Completed \\
\hline August & University IRB obtained and Community Partner Approved Action Research- August \\
\hline August & Community Partner and Agency identified possible At-Risk youth within their program- August \\
\hline August & $\begin{array}{l}\text { Parent Psychoeducational Meeting Held about ACES and Trauma Healing Club Intervention- August } \\
\text { - Parent Training on ACEs } \\
\text { - Parent Question \& Answer Session/ Brainstorming and Needs Assessment } \\
\text { - Parent Consent Obtained } \\
\text { - Parent Pre-test Assessments Administered }\end{array}$ \\
\hline August & $\begin{array}{l}\text { Student Psychoeducational Meetings Held about ACES and Trauma Healing Club Intervention- August } \\
\text { - Student Training on ACES } \\
\text { - Student Question \& Answer Session } \\
\text { - Student Assent Obtained } \\
\text { - Student Pre-test Assessments Administered }\end{array}$ \\
\hline September & $\begin{array}{l}\text { 12-week Intervention Started- September } \\
\text { - Students were screened every session with the SUDS assessment }\end{array}$ \\
\hline November & $\begin{array}{l}\text { Parent Follow Up Sessions Held to Discuss Needs as well as progress noted- November } \\
\text { - Parents completed social validity assessments } \\
\text { - Planning for the Trauma Healing Club celebration }\end{array}$ \\
\hline November & $\begin{array}{l}\text { Student Final Session- November } \\
\text { - Students completed Post-test assessments and social validity assessments } \\
\text { - Planning for the Trauma Healing Club Celebration }\end{array}$ \\
\hline December & Final Celebration Held and Peer Mentors Chosen to Assist in Future Trauma Healing Club Sessions-December \\
\hline January & Results of the Trauma Healing Club Intervention Shared with Stakeholders, Partners, Students and Parents-January \\
\hline February & Services re-started for second cohort \\
\hline
\end{tabular}

Experiences (ACEs) and the impact ACEs has on child development. Once the parent training was completed, parents were informed of the proposed 12-week trauma-based intervention, and parental consent was obtained.

Students were initially identified by the community refugee program partner based on needs related to behavioral concerns in their after-school program. The following data were tracked throughout the intervention to monitor effectiveness on decreasing trauma symptoms and to monitor an increase in participant awareness and ability to regulate. After the initial parent training, each student was then informed of their potential participation in the 12-week intervention and student assent was obtained. Following the assent obtainment, each student was asked to complete several assessment tools so we could better understand the level of need prior to intervention. These specific screenings were collected at both pre- and postintervention for purposes of outcome analysis. The assessments administered to the student participants were as follows: Child PTSD Symptom Scale (CPSS), Subjective Units of Distress (SUD) measures, and an adapted Child Intervention Rating Profile (CIRP). These assessments are discussed further under the instrument section of this manuscript.

\section{Instruments and Assessments Administered}

\section{Student Instruments and Assessments}

This research aimed to decrease trauma-related symptoms, increase effective coping skills, and increase student's ability to function. There instruments were used to identify inclusion criteria and to monitor student progress for the duration of the Trauma Healing Club Intervention.

Trauma Symptom reduction was obtained by a preand post-assessment using the Child PTSD Symptom Scale (CPSS) scale developed by Edna Foa and research by Foa et al. in (2001). The Child PTSD Symptom Scale (CPSS) is a DSM-IV criteria-based assessment and yields PTSD scores related to symptomology. The Likert-scale assessment measures PTSD symptom across domains of re-experiencing, avoidance, and hyperarousal. The CPSS comprises 24-items, 17 of which correspond to the DSMIV symptoms. Scores range from $0-7$, with higher scores indicating greater functional impairment. This assessment is administered at baseline (prior to the intervention to determine appropriateness for the group intervention and to determine levels of impairment), and the administered again after the last intervention session is concluded. 
Participants were chosen for this intervention if they scored a 14 or higher on the CPSS inventory.

Participant's ability to communicate and utilize learned skills to regulate emotions and feelings within the group and beyond is obtained using Subjective Units of Distress Scales (SUDS) or Feelings Thermometer assessment administered throughout the group intervention. The original Subjective Unit of Distress Scale was developed by Joseph Wolpe in (1969). A Subjective Units of Distress Scale (SUDS) is used to measure the intensity of distress or anxiety/ depression in people with impairments. The SUDS is a self-assessment tool rated on a scale from 0 to 10 . The SUDS is a subjective tool used by the clinician to evaluate student progress and the success of the student's current treatment and intervention. In this way, it can be used regularly over the months, or even in each session of your treatment to gauge different areas of disturbance that require additional work. The SUD scales are obtained pre- and post-for every intervention session. This is a common technique in cognitive therapy as a tool to gauge one's distress or emotional state.

To enhance the use of the Trauma Healing Club intervention, social validity measures were obtained from the participants. Student perceptions of the intervention were obtained through a pre- and post-intervention survey known as the Child Intervention Rating Profile (CIRP). The Child Intervention Rating Profile (CIRP) was adapted from the original Intervention Rating Profile (IRP) developed by researchers Witt and Martens in (1983). The IRP was adapted to work as a measure for this study to extend research in treatment acceptability to trauma treatments and to make practitioners more aware of interventions considered acceptable by student and child participants. This is subjective information obtained through student self-report about the students' perceptions of the intervention and how helpful it was. This form of assessment is known as a social validity. Social validity measures may help us to identify common features of procedures that are likely to be adopted and persist in a specific environment overtime by being deemed acceptable by the participant.

Children identified as in need from the original CPSS pretest scores participated in the Trauma Healing Club intervention. Students in need of more intensive services were referred for additional supports based on their progress in the 12-week intervention.

\section{Results}

The statistical analysis utilized for this research included pre- and post-analysis and t-tests of the obtained data. Data reflected on each of the following measures was used in this research to show the overall effectiveness of this intervention and treatment package.

\section{Results}

\section{CPSS}

The Child PTSD Symptom Scale (CPSS) is used to assess the participants level of reported PTSD symptomology before and after the intervention. The CPSS is also used to determine if a student meets the criteria for inclusion in the intervention. One hundred students who attended the community partner program were screened with the Child PTSD Symptom Scale (CPSS) to determine their appropriateness of participation in The Trauma Healing Club intervention. Of the 100 students screened a total of $\mathbf{8 8}(\mathbf{8 8} \%$ of these children) met the criteria for the intervention group based on life experiences and previous adverse childhood experiences and were offered the opportunity to participate. Of the 88 students who were offered the opportunity to participate in this research, $100 \%$ of them signed assents and were included in this research.

The following graph (Fig. 1) shows the average pre- and post-scores for the 88 students who participated in this intervention and treatment package. A score of 14 or higher on the CPSS indicates the need for clinical intervention. The average pre-test CPSS scores fall within the clinical range for need, and after the intervention the CPSS average scores show not only a significant improvement but CPSS scores well below the inclusion criteria score of 14 . The average CPSS score for the 88 participants was 27 at pretest; the average CPSS score for the 88 participants post intervention was a score of 8 . These outcomes represent a clinically significant improvement in participant reported PTSD symptomology from pre to post assessment.

\section{Subjective Units of Distress Scale (SUDS)}

Subjective units of distress (SUD) were obtained both pre- and post-intervention for each participant in every session across the 12-week intervention. There was an $85 \%$ completion rate for the pre-and post-session SUDS for all 88 participants across the 12-week intervention. Due to unanticipated absences of some participants across the 12-week intervention, we were unable to obtain $100 \%$ completion rate for the pre- and post-session SUDs for every participant; however, SUD scale findings were positive. Figure 2 below shows the average SUD scale reports for participants for each of the 12 sessions. It is evident from this data, that most of the participants displayed higher SUD 
Fig. 1 Pre and Posttest CPSS Scores
Average Pre and Posttest Scores on CPSS

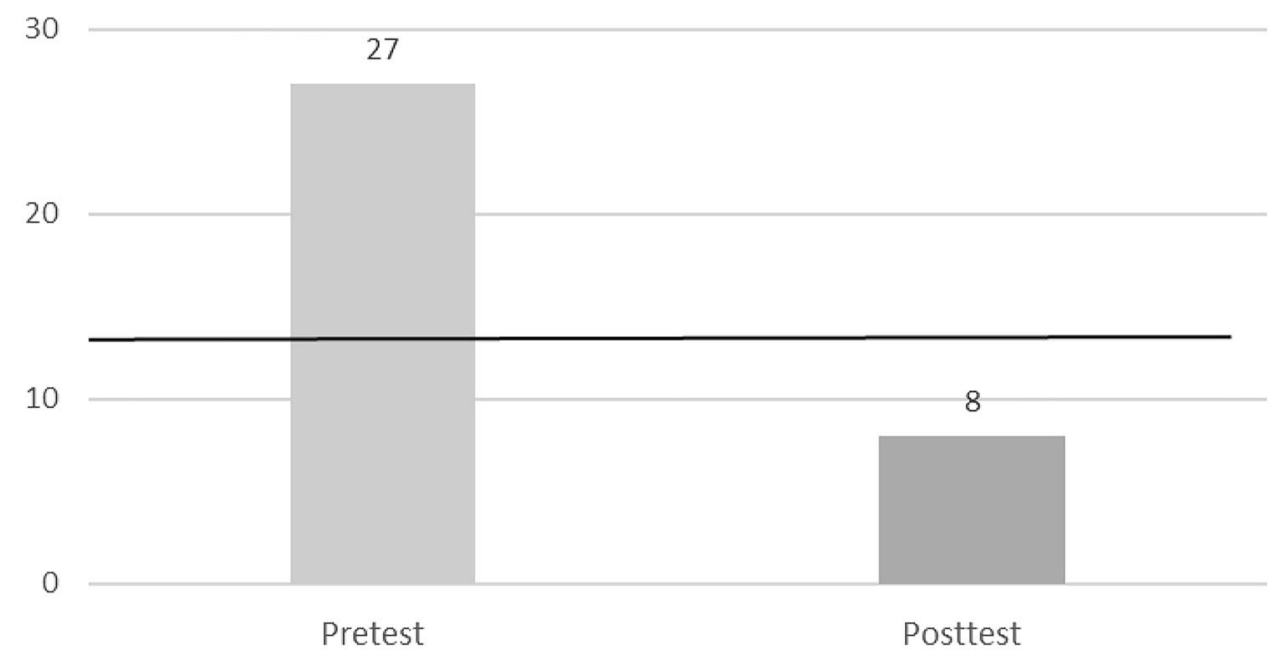

scale scores at the beginning of the intervention (Session 1 ), and as they moved through the program and received additional supports and coping skills- as well as exposure to processing their trauma within the group- their reported SUD scale scores decreased over time. This is data is right in line with the reported average pre and post CPSS outcomes that were previously discussed. Additionally, you can see a decreasing trend in the average reported SUDS across each session. There was a noticeable spike in reported SUDS pretest for Sessions 4 and 5, and the researchers attribute this to the participants beginning their individual trauma work and an increase in anxiety around that portion of the intervention; however, we continued to see a decrease in SUDS reports post session for every session across the 12 weeks.

\section{Child Intervention Rating Profile (CIRP)}

The CIRP asks the students questions about their perceptions of the intervention, and whether they will be impacted by the intervention and the way in which teachers or peers may perceive them. Initially, during the pretest, all respondents $(\boldsymbol{n}=\mathbf{8 8} ; \mathbf{1 0 0} \%)$ felt that the Trauma Healing Club intervention could be helpful,but they were also concerned that the intervention may negatively impact the way their teachers and peers perceive them; however, post-test data indicated that all $\mathbf{8 8}$ respondents $\mathbf{( 1 0 0 \% )}$ indicated that the intervention was very helpful, their peers and teachers were very supportive, and they would recommended the intervention to their peers. To
Fig. 2 Average Pre and Posttest SUDS Scores for Participants
Average Pre and Posttest SUDS Scores for

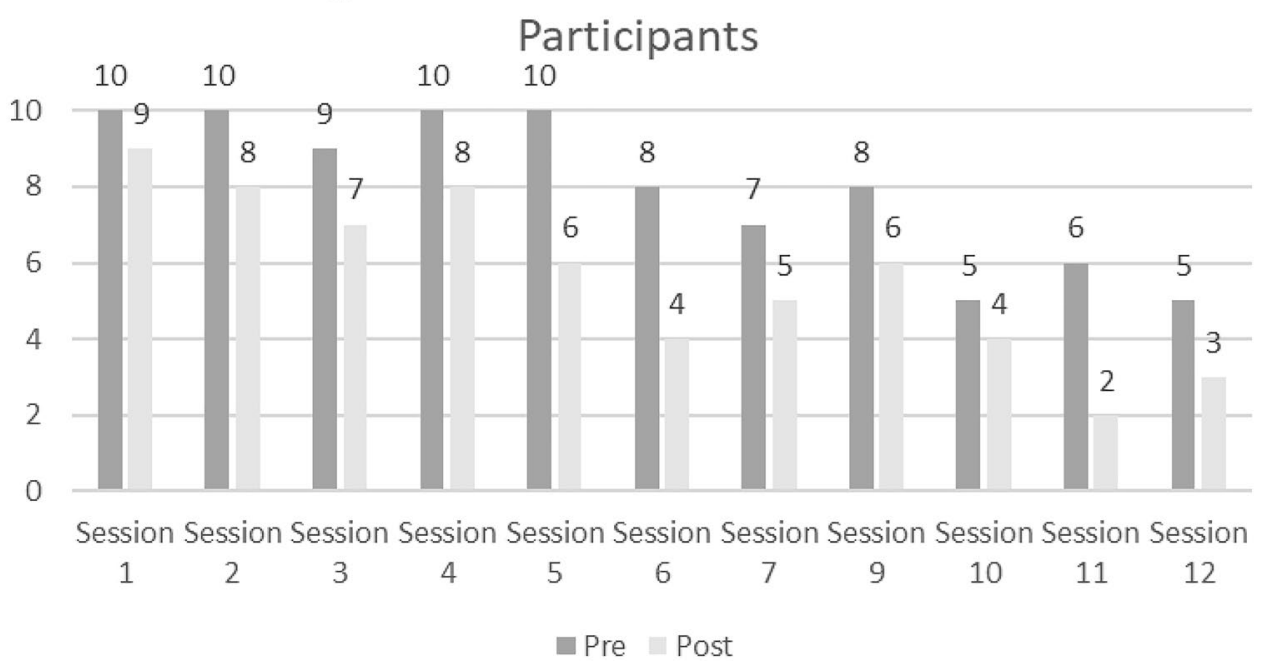


corroborate, a list of some of their anecdotal responses are included and are as follows:

"I liked being in this club with my friends. I gained friends from this club."

"I learned how to bring happiness and relaxing into my life."

"I enjoyed making my own beat because it made me

happy and to feel your own energy."

"I enjoyed the drumming because it took my stress away."

"I enjoyed the Trauma Healing Club because it was a lot of fun. The drumming was my

favorite because I got to learn new skills, I also got

to see friends who are far away from me."

These reported outcomes support the fact that the adapted CBITS intervention was successful in developing healthy coping skills within this population while also decreasing PTSD symptomology reported by the refugee student population. As previously referenced in Table 1, these minor changes, and edits to the CBITS curriculum provided culturally responsive supports for this population. Simple changes to the original CBITS curriculum ranged from extending the weeks of service delivery from 10 to 12 weeks, to simple terminology changes in the curriculum (i.e. from homework to independent project) that were considered more culturally supportive based on interactions and feedback from the participants. The African Drumming component was added to assist the students with feeling more comfortable with discussing the topic of trauma. Based on previous research it was indicated that embedding already existing cultural practices into and evidence-based intervention could assist with treatment and intervention acceptance and overall success ( $\mathrm{Li}$ et al., 2017). Additionally, culturally adapting an evidence-based practice is a systematic modification of an evidence-based treatment which takes into account language, terminology, cultural, and context in such a way that it is compatible with the client's cultural patterns, meaning, and values (Castro et al., 2010). We found this to be true with the inclusion of the drumming processes. Some of these adaptations were made prior to the intervention starting, and some were made as the program progressed based on responses from the participants.

\section{Limitations}

There were several study limitations including small sample size and limited access to multiple African refugee nations for participation in the study. The study did yield positive outcomes for the African refugee families who were served by the local refugee community-partner; however, more work is needed to determine the ability for this culturally responsive treatment package to be used across demographics and ethnic groups and larger sample sizes would benefit the research.

One limitation for this study was the small sample size utilized for this intervention. Small sample sizes often do not allow a proper representation of the larger population; however, because we were only able to access a smaller subset of the larger population of African refugees, only those found within one West Tennessee large urban area, a small sample size is expected for this specific minority population found in this research. A larger sampling of African refugees from across the nation would assist in determining the effectiveness of this sample intervention for a larger grouping of this ethnic population. Future researchers should focus efforts on African refugees across the nation to obtain a better sampling for this work.

\section{Conclusion}

This culturally adapted CBITS intervention was only implemented in one region housing the United States resettlement programs; these transitional resettlement programs often attempt to create a community of refugees from the same nation. However, such a cultural assumption fails to account for the arbitrary nature of African nation states created by colonizing European powers. An example of this is when the USA created a Sudanese community in a region, with 300 refugees, but the US failed to consider that within that group there were 13 distinct ethnic groups represented and each had their own culture and language (Fox \& Willis, 2009). This is indicative of the tendency the resettlement programs have in assuming that one nation means one culture; thus, all African refugees can be processed as though they come from the same culture (Fox $\&$ Willis, 2009). The researchers did their best to support the individual needs of all participants and incorporate their respective "community" and culture into the intervention and program; however, this small research study only represent a small portion of the refugee population found across the United States. More research is needed adapting this intervention to other refugee communities.

Future researchers should evaluate the effectiveness of this treatment package with larger samples size, with other African refugee and ethnic groups. This treatment package proved to be effective, but more research across ethnic groups is needed. Additionally, future researchers should investigate other adaptations to the intervention and treatment package such as adding in expressive arts activities, dance-movement activities, and even yoga programming. This research indicated that participants in a 
trauma-informed group intervention would benefit from the inclusion of expressive arts programming that is more reflective of their ethnic and cultural heritage.

\section{Declarations}

Conflict of Interest Statement The authors declare that they have no conflict of interest.

\section{References}

Adedoyin, A. C., Bobbie, C., Griffin, M., Adedoyin, O. O., Ahmad, M., Nobles, C., \& Neeland, K. (2016). Religious coping strategies among traumatized African refugees in the United States: A systematic review. Social Work \& Christianity, 43(1), 95-107.

Akinsulure-Smith, A. M. (2012). Using group work to rebuild family and community ties among displaced African men. The Journal for Specialists in Group Work, 37(2), 95-112.

Bensimon, M., Amir, D., \& Wolf, Y. (2008). Drumming through trauma: Music therapy with post-traumatic soldiers. The Arts in Psychotherapy, 35(1), 34-48. https://doi.org/10.1016/j.aip.2007. 09.002

Carlsson, F., Daruvala, D., \& Johansson-Stenman, O. (2005). Are people inequality-averse, or just risk-averse? Economica, 72(287), 375-396.

Castro, F. G., Barrera, M. Jr., Holleran Steiker, L. K. (2010). Issues and challenges in the design of culturally adapted evidencebased interventions. Annual Review Clinical Psychology, 6, 213239. https://doi.org/10.1146/annurev-clinpsy-033109-132032

Chinman, M., Oberman, R. S., Hanusa, B. H., Cohen, A. N., Salyers, M. P., Twamley, E. W., \& Young, A. S. (2015). A cluster randomized trial of adding peer specialists to intensive case management teams in the Veterans Health AdministraMon. Journal of Behavioral Health Services Research, 42, 109-121.

Chinman, M., George, P., Dougherty, R. H., Daniels, A. S., Ghose, S. S., Swi, A., \& Delphin-RiOmon, M. E. (2014). Peer support services for individuals with serious mental illnesses: Assessing the evidence. Psychiatric Services, 65, 429-441.

Clift, S. (2013). Optimism in the Field of Arts and Health. Perspectives in Public Health, 133(1), 1-18.

Cole, N. L. (2019). Understanding acculturation and why it happens. ThoughtCo. Retrieved August 6, 2020: https://www.thoughtco. com/acculturation-definition-3026039

Corrigan, P. W., \& Sokol, K. A. (2013). The impact of self-stigma and mutual help programs on the quality of life of people with serious mental illnesses. Community Mental Health Journal, 49, 1-6.

Delavega, E. (2020). Poverty fact sheets for west TN. Retrieved on 2/16/2021. https://www.memphis.edu/socialwork/research/files/ documents/2020povertyfactsheet_correct.pdf

Foa, E. B., Treadwell, K., Johnson, K., \& Feeny, N. (2001). Child PTSD symptom scale (CPSS): Validation of a measure for children with PTSD. Journal of Clinical Child Psychology, 30, 376-384.

Fancourt, D., Williamon, A., Carvalho, L. A., Steptoe, A., Dow, R., \& Lewis, I. (2016). Singing modulates mood, stress, cortisol, cytokine and neuropeptide activity in cancer patients and careers. Ecancermedicalscience, 10, 631. https://doi.org/10.3332/ecancer. 2016.631.

Fancourt, D., Perkins, R., Ascenso, S., Carvalho, L. A., Steptoe, A., \& Williamon, A. (2016). Effects of group drumming interventions on anxiety, depression, social resilience and inflammatory immune response among mental health service users. PLoS One, 11(e0151136), 1-16.

Faulkner, S., Wood, L., Ivery, P., \& Donovan, R. (2012). It is not just music and rhythm. Evaluation of a drumming-based intervention to improve the social wellbeing of alienated youth. Children Australia, 37, 31-36.

Filippi, M. K., Faseru, B., Baird, M., Ndikum-Moffor, F., Greiner, K. A., \& Daley, C. M. (2014). A pilot study of health priorities of Somalis living in Kansas City: Laying the groundwork for CBPR. Journal of Immigrant and Minority Health, 16(2), 314-320.

Fox, S. H., \& Willis, M. S. (2009). Initiatory mental health assessments for Dinka and Nuer refugees from Sudan. Journal of Immigrant \& Refugee Studies, 7(2), 159-179.

Goodkind, J. R., Hess, J. M., Isakson, B., LaNoue, M., Githinji, A., Roche, N., Vadnais, K., \& Parker, D. P. (2014). Reducing refugee mental health disparities: A community-based intervention to address postmigration stressors with African adults. Psychological Services, 11(3), 333-346. https://doi.org/10.1037/a0035081

Guenther, K. M., Pendaz, S., \& Makene, F. S. (2012). The impact of intersecting dimensions of inequality and identity on the racial status of eastern African immigrants. Sociological Forum., 26(1), 98-120. https://doi.org/10.1111/j.1573-7861.2010.01226.x

Hars, M., Herrmann, F. R., Gold, G., Rizzoli, R., \& Trombetti, A. (2014). Effect of music-based multitask training on cognition and mood in older adults. Age and Ageing, 43(2), 196-200.

Hays, T., \& Minichiello, V. (2005). The meaning of music in the lives of older people: A qualitative study. Psychology of Music, 33(4), 437-451.

Ho, F. N., Wang, H. M. D., \& Vitell, S. J. (2012). A global analysis of corporate social performance: The effects of cultural and geographic environments. Journal of business ethics, 107(4), 423-433.

Hooberman, J. B., Rosenfeld, B., Lhewa, D., Rasmussen, A., \& Keller, A. (2007). Classifying the torture experience of refugees living in the United States. Journal of Interpersonal Violence, 22, 108-123. https://doi.org/10.1177/0886260506294999

Jaranson, J. M., Butcher, J., Halcon, L., Johnson, D. R., Robertson, C., Savik, K., Spring, M., \& Westermeyer, J. (2004). Somali and Oromo refugees: Correlates of torture and trauma history. American Journal of Public Health, 94(4), 591-598.

Jaycox, L. H., Kataoka, S. H., Stein, B. D., Langley, A. K., \& Wong, M. (2012). Cognitive behavioral intervention for trauma in schools. Journal of Applied School Psychology, 28(3), 239-255. https:// doi.org/10.1080/15377903.2012.695766.

Johnson, Y. M., \& Munch, S. (2009). Fundamental contradictions in cultural competence. Social Work, 54(3), 220-231.

Kohli, H. K., Huber, R., \& Faul, A. C. (2010). Historical and theoretical development of culturally competent social work practice. Journal of Teaching in Social Work, 30(1), 252-271.

Kontak, D. (2012). School social work services. School Social Work Association of America. Sumner, WA: School Social Work Association of America. Retrieved from http://sswaa.org/associations/ 13190/files/School\%20Social\%20Work\%20Services\%20One\% 20Page\%202012.pdf

Li, W., Zhang, L., Luo, X., et al. (2017). A qualitative study to explore views of patients', carers' and mental health professionals' to inform cultural adaptation of CBT for psychosis (CBTp) in China. BMC Psychiatry, 17, 131. https://doi.org/10.1186/ s12888-017-1290-6

Matarasso, F. (1997). Use or ornament: the social impact of participation in the arts. Stroud: Comedia.

Matheson, K., Jorden, S., \& Anisman, H. (2008). Relations between trauma experiences and psychological, physical and neuroendocrine functioning among Somali refugees: Mediating role of coping with acculturation stressors. Journal of Immigrant 
and Minority Health, 10(4), 291-304. https://doi.org/10.1007/ s10903-007-9086-2

Meléndez Guevara, A. M., Lindstrom Johnson, S., Elam, K. et al. (2021) Culturally Responsive Trauma-Informed Services: A Multilevel Perspective from Practitioners Serving Latinx Children and Families. Community Mental Health J 57, 325-339.https://doi. org/10.1007/s10597-020-00651-2

Melton, C. The Sycamore Institute's analysis of the U.S. Centers for Disease Control and Prevention's (CDC) 2014-2017 Behavioral Risk Factor Surveillance System Data. Provided by the Tennessee Department of Health's Division of Policy, Planning and Assessment, Office of Health Statistics. [Online] Public use data retrieved from https://www.cdc.gov/brfss/annual_data/annual_ data.htm

Miller, K. E., \& Rasmussen, A. (2010). War exposure, daily stressors, and mental health in conflict and post-conflict settings: Bridging the divide between trauma-focused and psychosocial frameworks. Social Science \& Medicine., 70(1), 7-16.

Pearce, E., Launay, J., Machin, A., \& Dunbar, R. I. (2016). Is Group Singing Special? Health, Well-Being and Social Bonds in Community-Based Adult Education Classes. Journal of Community \& Applied Social Psychology, 26(6), 518-533. https:// doi.org/10.1002/casp. 2278

Ramsden, R., \& Taket, A. (2013). Social capital and Somali families in Australia. Journal of International Migration and Integration, 14(1), 99-117.

Reagon, C., Gale, N. S., Dow, R., Lewis, I., \& Van Deursen, R. (2016). A mixed-method systematic review to investigate the effect of group singing onhealth related quality of Life. Complementary Therapies in Medicine, 27(1), 1-11.

Richman, B. (1993). On the evolution of speech: singing as the middle term. Current Anthropology, 34(5), 721-722.

Rousseau, C., Mekki-Berrada, A., \& Moreau, S. (2001). Trauma and extended separation from family among Latin American and African refugees in Montreal. Psychiatry: Interpersonal and Biological Processes, 64, 40-59. https://doi.org/10.1521/psyc.64.1.40.18238.

Schwartz, S. J., Unger, J. B., Zamboanga, B. L., \& Szapocznik, J. (2010). Rethinking the concept of acculturation: Implications for theory and research. The American Psychologist, 65(4), 237-251. https://doi.org/10.1037/a0019330

The Urban Child Institute. (2013). Data Book on the State of Children in Memphis. Retrieved on 2/16/2021. http://www.urbanchildinstitute.org/ sites/all/files/databooks/TUCI_Data_Book_VIII_2013.00_complete. pdf.

Tennessee Health Department. (2017). Tennessee ACEs Final Report. Retrieved on 2/16/2021. https://www.tn.gov/content/ dam/tn/health/documents/Tennessee_ACE_Final_Report_with_ Authorization.pdf.
Trepasso-Grullon, E. (2012). Differences among ethnic groups in trauma type and PTSD symptom severity. Graduate Student Journal of Psychology, 14, 102-112.

UNHCR. (2019). Global Trends: Forced Displacement in 2019. Geneva: UNHCR. Retrieved on 6/29/2021: https://www.unhcr. org/flagship-reports/globaltrends/globaltrends2019/

UNHCR. (2020). Forced displacement passes 80 million by mid-2020 as COVID-19 tests refugee protection globally. Retrieved on 6/29/2021: https://www.unhcr.org/news/press/2020/12/5fcf94a04/ forced-displacement-passes-80-million-mid-2020-covid-19-testsrefugee-protection.html

U.S. Centers for Disease Control and Prevention (CDC). (2017). The behavioral risk factor surveillance system: complex sampling weights and preparing 2017 BRFSS module data for analysis. [Online] July 2018. https://www.cdc.gov/brfss/annual_data/2017/ pdf/Complex-Smple-Weights-Prep-Module-Data-Analysis-2017508.pdf

Vinesett, A. L., Price, M. \& Wilson, K. H. (2015). The journal of alternative and complementary medicine. Volume 21 , Number 8,2015 , pp. 460-465. https://doi.org/10.1089/acm.2014.0247

Washington, G., Johnson, T., Jones, J., \& Langs, S. (2007). AfricanAmerican boys in relative care: a culturally centered group mentoring approach. Journal of Social Work in Groups, 30(1), 45-69.

Washington, G., Barnes, D., \& Watts, R. J. (2014). Reducing risk with pyramid mentoring: a proposal for a culturally-centered group intervention. Journal of Human Behavior and the Social Environment, 24(6), 646-657.

Weinstein, D., Launay, J., Pearce, E., Dunbar, R. I., \& Stewart, L. (2016). Singing and social bonding: changes in connectivity and pain threshold as a function of group size. Evolution and Human Behavior, 37(2), 152-158.

Whaley, A. L., \& Davis, K. E. (2007). Cultural competence and evidence-based practice in mental health services: A complementary perspective. American Psychologist, 62(6), 563-574. https://doi. org/10.1037/0003-066x.62.6.563

Witt, J. C., \& Martens, B. (1983). Assessing the acceptability of behavioral interventions used in classrooms. Psychology in the Schools, 20, 510-517.

Wolpe, J. (1969). The practice of behavior therapy pergamon press 0080065635.

Yeager, K. A., \& Bauer-Wu, S. (2013). Cultural humility: Essential foundation for clinical researchers. Applied Nursing Research, 26(4), 251-256. https://doi.org/10.1016/j.apnr.2013.06.008

Publisher's Note Springer Nature remains neutral with regard to jurisdictional claims in published maps and institutional affiliations. 